\title{
A CASE OF ACONITE POISONING.
}

By c. G. Carleton, M. D., LAwrence.

Aт four o'clock A. M., June 6, 1879, I saw Mrs. S., a widow, age twenty-three, who had been under the care of a physician for so-called Bright's disease. I learned that at midnight she had taken from a trunk, in a dimly-lighted room, a bottle which she supposed contained spts. ether nit., and had poured without measurement into water an amount which she intended to be a teaspoonful. This she had drunk, and then fallen asleep, to be awakened at half past three by pain and general distress. The bottle from which the medicine had been poured was shown me, and was labeled "Tinct. Aconite, Poison," and subsequent inquiry at the druggist's proved it to be from the root. From the best judgment I could form the amount taken was about three drachms. At this time the patient was in bed complaining of extreme pain "all over," and "tingling of the veins." She was in pretty constant motion, throwing herself about the bed, and at times her legs off its sides, and there was a limited amount of spasmodic action; pulse 32, very feeble, wavy, and irregularly intermittent; the respirations were feeble, shallow, and irregular; pupils contracted; no special thirst or dryness of fauces, and no headache; the intellect was clear. A small amount of magnesia had already been given her.

Free emesis was induced by titillation of the fauces, and promoted by copious dranghts of warm water. A considerable quantity of undigested food was vomited at first, and afterwards bile and mucus. At $4.50 \mathrm{~A}$. M., her condition at this time being much the same, with the additional symptoms of profuse cold sweating, cold extremities, and constant screams with appeals for relief, I prepared fifteen drops of tincture of digitalis with a few drops of warm water, and gave her one half the amount by hypodermic injection in the fore-arm, and in ten minutes the remainder. These injections were followed at once by quite decided increase in the volume of the pulse, with a slight increase in its frequency. Meantime frictions with warmth were kept up, and a sinapism was applied over the epigastrium. At $5.20 \mathrm{~A}$. M. another hypodermic injection of fifteen drops of tincture of digitalis was administered. I then left her with directions that the head be kept low and frictions continued. At the earnest request of her mother she was permitted to inhale a few drops of "chloric ether," and this produced some quiet. Returning at 6.10 A. M., I found her condition slightly improved, - less sweating and less restlessness; but in reply to the inquiry if the pain were less, she shook her head in the negative, and constantly asked for relief. The pulse was but little better than at the beginning of treatment. I then gave another hypodermic injec- 
tion of fifteen drops of the tincture of digitalis, and the stomach, which until now had been frequently expelling mucus, being somewhat quiet, I gave a mixture of brandy and ammonia, which, however, was quickly rejected. Sips of brandy were subsequently given at frequent intervals, but not retained. As there had been no dejection for a week, and as the attendants were hardly able to render the assistance which was imperatively demanded, I was unable to use stimulating enemata. I left her at 6.45 A. M., feeling that she was improving; the skin had more warmth, there was less sweating, and the pulse had increased to 42, with less frequent intermissions. There was also less restlessness, although the patient did not admit feeling any better. At $7.30 \mathrm{~A} . \mathbf{M}$. I found her sleeping, and she was with difficulty aroused, when she spoke feebly; the skin was warm and dry, and the extremities warm; still inclined to vomit if disturbed; pulse 44. I then gave another and final injection of fifteen drops of digitalis. At $9.30 \mathrm{~A}$. M. the pulse had increased to 54 , and at $11.30 \mathrm{~A}$. M. to $7 \%$, with the action of the heart normal. The vomiting gradually ceased, brandy, and later other nourishment, was retained, and her subsequent improvement was uninterrupted. Upon the following day I found an erythematous blush upon the arm about the punctures of the needle, accompanied by some pain; but this at my last visit, the third day, was passing away. At each visit she spoke of "tingling," which upon the third day was very slight. The result of the treatment in this case seems to me to reinforce very strongly the arguments which were, I believe, first suggested by Dr. J. Milner Fothergill in $\mathbf{1 8 7 1}$ for the physiological antagonism between digitalis and aconite, and which were, so far as I am able to learn, first applied in the human subject by Dobie in a case reported in the British Medical Journal in December, 1872.

\section{$\longrightarrow$ \\ RECENT PROGRESS IN ORTHOPEDIC SURGERY. ${ }^{1}$}

BY E. H. BRADFORD, M. D.

Pathology of Joint Diseases. ${ }^{1}$ - Volkmann's monograph illustrates that lip-joint disease, tumor albus, caries of the joint, are terms which convey to modern surgical pathologists a different meaning from that understood by the older writers.

Volkmann claims that his numerous resections have enabled him to study the disease at a stage not investigated before. He believes that fungous inflammations of the joint begin usually, and in children always, not as an arthritis (that is, in the joint), but as a localized ostitis, limited to a spot in the epiphysis next the joint. This is the condition in the

1 Sammlung klinischer Vorträge, 168, 169. Charakter und Bedeutung der fungösen Gelenkentzundungen. 